\title{
Artigo original \\ Estabilidade locomotora durante a condução de um cão
}

\author{
Bruna Carla Périco \\ Eliane Mauerberg-deCastro \\ Mayara Borkowske Pestana \\ Larissa Agnes Porto \\ Fernanda Lopes Magre \\ Stephanie C. Miranda Pacheco \\ Universidade Estadual Paulista
}

\begin{abstract}
Resumo-O objetivo deste trabalho foi avaliar a influência da percepção háptica na estabilidade locomotora do ser humano durante a condução de um cão com uma guia. Adultos ( $n=14)$, com e sem restrição da visão, andaram sobre uma trave de equilíbrio sozinhos, ou, com uma guia, andaram conduzindo um cão sobre um banco estreito ao lado. Quando os participantes caminharam conduzindo o cão, privados da visão, o desempenho locomotor-avaliado através da duração do deslocamento total, da duração das passadas, das fases de duplo suporte e balanço, do comprimento e velocidade das passadas, assim como da variabilidade da fase relativa-melhorou significativamente $(p \leq 0,05)$ em relação ao desempenho locomotor na condição sem o cão. Nas condições com visão, conduzir o cão não resultou em mudanças nestas variáveis. Concluímos que estes indivíduos foram capazes de utilizar a guia do cão para detectar propriedades hápticas com propósito de melhorar a estabilidade na locomoção.
\end{abstract}

Palavras-chaves: locomoção, terapia assistida por animais, cão

\begin{abstract}
Locomotion stability while dog walking.” The purpose of this study was to evaluate how walking a dog on a leash influenced haptic perception and stability during human locomotion. Fourteen adults, with and without sight restriction, walked on a narrow balance beam by themselves, or holding a leash attached to a dog that walked on a parallel narrow bench. The performance of participants who walked with the dog, but blindfolded, significantly $(p \leq 0.05)$ improved as compared to the condition without the dog. Their locomotion performance was assessed via the duration of the total displacement on the balance beam, durations of the double support phase, swing phase, stepping cycle, stepping length, and linear velocity, as well as variability of the inter-limb relative phase. With vision, these parameters were not affected by the presence of the dog. We conclude that these adults were able to use the dog's leash to detect haptic properties in order to achieve postural control (e.g., stability in speed performance) while walking.
\end{abstract}

Keywords: locomotion, animal assisted therapy, dog

Resumen- “La estabilidad locomotora durante la conducción de un perro.” El objetivo de este estudio fue evaluar la influencia de la percepción háptica en la estabilidad del aparato locomotor humano, mientras que la conducción de un perro con una guía. Los adultos $(\mathrm{n}=14)$, con y sin restricción de la visión, caminaron sobre una barra de equilibrio solo, o simultáneamente con el perro en la guia en un banco al lado. Cuando participantes caminaron con el perro, privados de vista, el rendimiento locomotor-evaluado por la longitud total de desplazamiento, la longitud de los pasos, las fases de doble apoyo e impulsión, la longitud y la velocidad del pasado, así como la variabilidad de la fase relativa-mejoró significativamente $(p \leq 0.05)$ en comparación con el rendimiento locomotor en la condición sin el perro. En las condiciones con la visión, impulsar el perro no dio lugar a cambios en estas variables. Llegamos a la conclusión de que estos individuos fueron capaces de utilizar la guía del perro para detectar propiedades hápticas con el propósito de mejorar la estabilidad en la locomoción.

Palabras claves: locomoción, terapia asistida por animales, perro

\section{Introdução}

O vínculo com animais está cada vez mais presente no cotidiano do ser humano, seja no âmbito pessoal, profissional e, mais especificamente, no âmbito terapêutico. Por exemplo, cães são animais bastante populares tanto como animais de serviço e suporte à vida diária de pessoas com deficiência ou necessidades especiais, assim como durante intervenções terapêuticas. Em geral, pesquisas buscam avaliar os efeitos dessas relações no domínio afetivo (Banks \& Banks, 2002; Martin \& Farnum, 2002; Serpell, 2004; Tardona 2012), no domínio social (Carlisle, 2012; Cohen, 2004; 
Grandgeorge, Tordjman, Lazartigues, Lemonnier, Deleau, \& Hausberger, 2012; Kushner, Blatner, Jewell, \& Rudloff, 2006, Toohey \& Rock, 2001), na aprendizagem (Capote, 2009; Jalongo, Astorino, \& Bomboy, 2004; Katcher \& Teumer, 2006), no domínio físico e do comportamento motor (Cutt, GilesCorti, Knuiman, \& Pikora, 2008; Friedman, Thomas, Stein, \& Kleiger 2003; Gee, Harris, \& Johnson, 2007; Johnson \& Meadows, 2010). Por exemplo, no campo do comportamento motor, temos estudos mostrando a estreita relação adaptativa do sistema postural durante o manejo ou contato com outro animal. Um popular exemplo é o uso do cavalo como instrumento cinesiotera-pêutico na reabilitação de problemas de controle postural (e.g., indivíduos com paralisia cerebral). Menos populares são estudos analisando o resultado da interação entre um ser humano e um cão na função postural. Indivíduos cegos que usam um cão como guia geralmente se beneficiam navegando eficientemente pelo espaço.

Alguns estudos demonstraram que o manejo de um cão através da guia transmite informação háptica ${ }^{1}$ e auxilia na orientação postural e na mobilidade de adultos privados da visão (Mauerberg-deCastro, Moraes, Tavares, Pestana, Porto, \& Dias, 2013; Melo, 2011), de deficientes intelectuais (Pestana, Mauerberg-deCastro, Périco, \& Porto, 2012) e de cegos (Zabihaylo, Couturier, Termoz, \& Prince, 2005).

A guia do cão, tal como a rédea do cavalo, é uma ferramenta não rígida que transmite informação háptica sobre os movimentos destes, e pode, por sua vez, exercer influências no movimento do condutor que, supostamente, controla o animal.

Tendo em vista evidências experimentais do papel da percepção háptica durante o manejo de ferramentas não rígidas, Mauerberg-deCastro (2004) desenvolveu um modelo experimental chamado sistema "âncora" para ilustrar o papel do sistema háptico no controle postural. O sistema "âncora" é uma ferramenta manipulada pelo executante com o objetivo de melhorar a orientação de seu corpo em tarefas posturais desafiadoras. Em situação experimental, o sistema âncora inclui a manipulação de cabos flexíveis seguros um em cada mão, com cargas presas em cada extremidade dos cabos e mantidas esticadas, porém, ao mesmo tempo, sempre em

\footnotetext{
${ }^{1}$ A clássica definição de percepção háptica (toque ativo ou toque háptico) resulta da estimulação de mecanorreceptores-na pele, músculos, tendões e articulações—pela exploração de um objeto no espaço (Gibson, 1962). A percepção háptica permite-nos, por exemplo, identificar um objeto, ou alguma de suas características (seu tamanho, forma, peso, sua posição, ou localização) a partir do contato com a fonte que contém a informação alvo da exploração. O toque háptico resulta de esforço muscular e as consequências sensoriais. O sistema háptico pode, por exemplo, através de elementos não-biológicos (i.e., ferramentas) anexos ao corpo expandir referências sobre o meio e detectar informação distais ao ponto de contato biológico (Burton, 1993). Por exemplo, um cego com uma bengala amplia sua noção de orientação espacial, assim como um indivíduo com dificuldades posturais durante a marcha pode estabilizar sua postura com leves contatos em superfícies de apoio (Gentaz, Baud-Bovy \& Luyat, 2008; Mauerberg-deCastro, 2004).
}

contato constante com a superfície.

Vários estudos integrando o sistema âncora em tarefas com restrição ao equilíbrio corroboraram efeitos estabilizadores da postura em adultos (Mauerberg-deCastro, 2004), idosos (Dascal, Okazaki, \& Mauerberg-deCastro, 2012; Moraes \& Mauerberg-deCastro, 2009; Freitas, MauerbergdeCastro, \& Moraes, 2013), crianças (Calve \& MauerbergdeCastro, 2005) e em jovens e adultos com deficiência intelectual (Mauerberg-deCastro, Moraes, \& Campbell, 2012; Mauerberg-deCastro, Lucena, Cuba, Boni, Campbell, \& Moraes, 2010; Polanczyk \& Mauerberg-deCastro, 2005). Esses efeitos estabilizadores são mais significativos quando a demanda da tarefa postural é maior (e.g., equilibrar-se sob uma privação visual).

A redução da oscilação e melhora da estabilidade corporal pode ser encarada como produto da interação toqueancoragem. Com a utilização do toque, a informação visual pode não ser a fonte predominante para o controle postural, mas a informação integrada de um conjunto de outros sistemas sensoriais que resulta em eficiência e controle ao sistema postural (Bonfim, Polastri, \& Barela, 2006). Ainda, sem o uso do toque, a informação visual é a fonte primordial para manutenção da orientação e estabilidade do corpo.

Mauerberg-deCastro e seus colaboradores expandiram o conceito do sistema âncora para ilustrar mecanismos hápticos durante a condução e o manejo de um cão (Mauerberg-deCastro et al., 2013; Melo, 2011; Pestana et al., 2012;). Similar aos resultados de outros estudos sobre o sistema âncora, efeitos estabilizadores na postura do ser humano foram observados durante a condução do cão. Diferente do sistema âncora originalmente proposto—onde um sistema biológico integra dados do meio utilizando-se de uma extensão "ancorada" num ponto terminal estático (i.e., a superfície)—, durante a tarefa de condução de um cão, dois sistemas biológicos (cão e ser humano), acoplados através da guia (i.e., sistema âncora), influenciam um ao outro alterando seus níveis de estabilidade postural e de orientação.

Assim, a questão deste estudo é: num contexto onde dois sistemas biológicos estão ancorados entre si por uma ferramenta (i.e., uma guia), pode o ser humano, exposto a uma situação de desafio ao equilíbrio, encontrar informação háptica útil a ponto de estabilizar sua postura durante uma tarefa locomotora? Nossa predição é de que o movimento corporal do cão, embora potencialmente perturbador ao condutor, também serve como fonte de orientação (através da guia) quando uma situação crítica (e.g., ameaça de uma queda) se apresenta. Nesta situação, é possível que o condutor module a tensão na guia não mais para controlar o animal, mas para obter (momentaneamente) dados de posição do próprio corpo tomados com base nas reações posturais desencadeadas no animal. Por outro lado, se o movimento do cão inadvertidamente adicionar influências desestabilizadoras ao movimento do seu condutor, é possível que a dupla tarefa háptica (i.e., controlar o cão e extrair dados sobre a posição do próprio corpo a partir das reações posturais do cão) seja incompatível ao sistema de controle motor e ambas 
compitam entre si, levando a maior desequilíbrio quando o requerimento da tarefa for extrapolado da situação normal da locomoção (e.g., potencial de queda). Neste caso, o controle do animal com a guia é abandonado para dar lugar a uma necessidade mais emergencial, a de não cair.

O presente estudo propôs confirmar resultados de Melo (2011) que observou participantes privados da visão conduzindo um cão sobre uma trave de equilíbrio exibirem melhora no controle postural durante o percurso. Através de variáveis descritivas do andar, o presente estudo avaliou a estabilidade locomotora durante a condução de um cão em tarefas de desafio ao equilíbrio.

\section{Método}

\section{Participantes}

Participaram do estudo 14 voluntários (oito do sexo feminino e seis do sexo masculino), com idade média de 24,14 $( \pm 2,89)$, sem alterações musculoesqueléticas e neurológicas. Dentre os participantes, nenhum deles tinha experiência prévia de adestramento de cães.

Também participou do estudo uma fêmea canina da raça Akita com três anos de idade, 35 quilos e com altura na cernelha de $68 \mathrm{~cm}$, previamente preparada para a tarefa do estudo. O animal teve como pré-requisitos: ser sociável, não possuir histórico de agressividade e receber treinamento em comandos básicos de obediência (Becker, 2011). O estudo foi executado sob a aprovação do Comitê de Ética em Pesquisa com seres humanos (UNESP/IB/CEP n 1024/2012) e com animais (UNESP/IB/CEA n 1841/2012).

\section{Materiais e instrumentos}

Uma trave de equilíbrio foi posicionada paralelamente a $40 \mathrm{~cm}$ de um banco sueco. A trave media 34 centímetros $(\mathrm{cm})$ de altura, $10 \mathrm{~cm}$ de largura e 6 metros de comprimento, e o banco media $34 \mathrm{~cm}$ de altura, $28 \mathrm{~cm}$ de largura e 6 metros de comprimento; duas câmeras da marca Panasonic PV-GS300 com capacidade de registro de 60 quadros por segundo; um sistema de referência para calibração do espaço da tarefa composto por 8 cabos flexíveis com 3 marcadores em cada um deles, 8 fios de prumo; 3 marcadores de pontos anatômicos confeccionados com velcro; bolas de isopor de $2 \mathrm{~cm}$ cada e fita reflexiva de cor branca; duas guias de $100 \mathrm{~cm}$ (uma guia de manejo pelo participante e outra de segurança manejada por um auxiliar); uma venda para os olhos de cor preta.

A calibração do espaço de coleta foi realizada por meio de uma referência com 24 pontos distribuídos em 8 cabos flexíveis que foram colocados cada um em um gancho fixado ao teto da sala formando um corredor entre os cabos. Neles foram fixados três marcadores na direção vertical (comprimento do fio) com intervalo de 1 metro entre eles. Para manter o cabo estático, foi colocado um prumo em sua ponta, próximo ao chão.
Foram realizadas marcações nos seguintes pontos anatômicos do lado esquerdo do participante: ombro (projeção do processo acromial da escápula) e quadril (projeção do trocânter maior do fêmur) e pé (calcâneo). Essa marcação permitiu rastrear a trajetória desses pontos nas direções das coordenadas "x", "y" e "z". As marcações foram feitas com bolas de isopor, recobertas por fita reflexiva de cor branca. Neste estudo foi apenas utilizada a informação dos pontos do tornozelo e quadril para determinação das variáveis em análise (ver abaixo).

As imagens foram filmadas pelas duas câmeras, com distância de 4 metros entre elas, e 6 metros da trave de equilíbrio de modo a visualizar a extensão toda da trave e do banco. As imagens filmadas pelas duas câmeras durante as coletas foram capturadas pela placa de captura de imagens da marca Pinnacle Studio DV, versão 10.5 em arquivos com formato AVI (audio video interleave). As imagens desses arquivos foram processadas utilizando o programa Digital Video for Biomechanics for Windows 32 bits (Dvideow) versão 5.1" (Figueroa; Leite \& Barros, 2003).

\section{Procedimento}

A tarefa dos participantes consistia em andar sobre uma trave de equilíbrio com e sem a condução do cão e com e sem o uso da visão em quatro condições experimentais: andar de forma independente e sem auxílio, com visão (SCCV); andar conduzindo o cão, com visão (CCCV); andar de forma independente e sem auxílio, sem visão (SCSV); e andar conduzindo o cão, sem visão (CCSV). Foram realizadas seis tentativas em cada condição. A ordem de realização das condições experimentais e respectivas tentativas foi completamente randomizada.

A tarefa foi controlada por dois experimentadores, sendo que um deles ficou posicionado atrás do participante para servir de segurança no evento de uma queda, e o outro, posicionado ao lado do banco onde o cão se deslocava. O experimentador que controlava a participação do cão deslocava ao lado do cão, segurando uma guia adicional, de modo a monitorar sua atividade (no caso do mesmo avançar à frente do participante, parar ou tentar sair da área de deslocamento sobre o banco).

Em todas as condições foi dada instrução ao participante para que, enquanto conduzisse o cão, ele caminhasse na trave da maneira mais natural possível. A tarefa iniciava a partir do comando verbal de um dos experimentadores que ficava posicionado mais próximo ao participante.

Nas condições sem o uso da visão, foi colocada uma venda nos olhos do participante quando ele estava em cima da trave antes do início da tentativa. Nestas condições, o participante era verbalmente informado sobre o final do percurso na trave no início do último passo.

\section{Análise dos dados}

A visualização quadro a quadro das imagens no progra- 
ma Dvideow versão 5.1 permitiu o registro manual dos quadros nos quais ocorreram os eventos da marcha (primeiro toque do calcanhar direito, segundo toque do calcanhar direito, toque do calcanhar esquerdo, perda de contato do pé direito, perda de contato do pé esquerdo) e da posição da coordenada $\mathrm{x}$ no toque de calcanhar dos pés na trave durante o percurso. O registro desses dados foi realizado no programa Microsoft Excel que permitiu calcular as variáveis: duração dos ciclos da passada, duração da fase de balanço e do duplo suporte, comprimento da passada e velocidade da passada. Para os cálculos dessas variáveis foram excluídos o primeiro e o último ciclo da marcha em todas as tentativas realizadas de modo a evitar as influências de aceleração/desaceleração típicas no início e parada da locomoção.

As variáveis relacionadas à estabilidade locomotora durante $o$ andar do participante foram:

- Duração (em segundos) do deslocamento total sobre a trave: incluiu a diferença temporal (entre os quadros filmados) no momento inicial do andar e final (determinada pelo $1^{\circ}$ contato do calcanhar de um dos pés na trave, excluídos o ciclo inicial e final).

- Duração (em segundos) da fase de balanço: diferença temporal da ocorrência da perda de contato dos dedos dos pés da trave e o próximo toque de calcanhar do mesmo pé na trave.

- Duração (em segundos) do duplo suporte: período em que ambos os pés permanecem em contato com a trave.

- Duração (em segundos) ou ciclo das passadas: diferença temporal entre o $1^{\circ}$ e $2^{\circ}$ contatos do calcanhar do mesmo pé com a trave de equilíbrio.

- Comprimento da passada: incluiu a diferença entre o valor da coordenada x no momento do contato inicial do pé na trave de equilíbrio e o valor da coordenada x no próximo contato do mesmo pé, e assim por diante. Um sistema de referência para calibração foi inserido ao longo de todo o comprimento da trave com intervalos de 1 metro cada. Este sistema permitiu computar o valor em centímetros do comprimento das passadas.

- Velocidade linear das passadas (cm/s): calculada através da divisão do comprimento da passada pela duração da passada.

- Fase relativa intermembros: relação temporal relativa entre os membros inferiores, direito e esquerdo. A fase relativa foi calculada através da subtração do tempo de ocorrência do toque de calcanhar direto e o tempo de ocorrência do toque de calcanhar contralateral dividido pelo tempo total de duração da passada. Esta variável permite avaliar aspectos temporais da coordenação entre membros (Mauerberg-deCastro \& Angulo-Kinzler, 2001). Quando a coordenação é estável, a somatória dos valores da fase relativa dos dois membros resulta em um valor próximo de 1,0. Para cada membro, a medida da fase temporal relativa próxima de 0,5 indica simetria e é influenciada pela estabilidade postural. O desvio-padrão da fase relativa permitiu avaliar, assim como as demais variáveis descritas acima, o nível de estabilidade locomotora.

\section{Análise estatística}

Foram realizadas análises de variância (ANOVA) com medidas repetidas nos fatores visão (sem e com visão), condição da tarefa (sem e com cão) e seis tentativas para as variáveis: deslocamento total na trave, durações do duplo suporte, da fase de balanço, e da passada, comprimento das passadas, e velocidade linear das passadas, fase relativa intermembros (valores médios e de desvio-padrão de cada tentativa).

Testes a posteriori de Bonferroni foram utilizados para as comparações aos pares quando efeitos foram detectados. O valor de significância estatística foi mantido em á 0,05 . Quando os efeitos principais ou de interação resultaram em diferenças significativas, foi então calculado o tamanho do efeito usando o parâmetro ETA ao quadrado $\left(\eta^{2}\right)$. De acordo com Thalheimer e Cook (2002), um tamanho de efeito de 0,8 é grande, 0,5 é médio ou moderado, e 0,2 é pequeno.

\section{Resultados}

Neste estudo, o nível de estabilidade locomotora em função da manipulação das tarefas foi inferido através de diversas variáveis temporais e espaciais, cujos resultados são descritos abaixo. Para cada variável, valores médios de cada tentativa foram computados para a análise estatística. Da fase relativa intermembros, incluímos, além dos valores da média, os valores de desvio-padrão.

\section{Duração do deslocamento total}

Os valores da média e desvio-padrão calculados a partir das seis tentativas do deslocamento total de cada condição experimental foram: 6,16 segundos $(\mathrm{s})( \pm 0,3)$ na condição SCCV; 7,58s $( \pm 0,31)$ na condição CCCV; 30,87s $( \pm 5,97)$ na condição SCSV e 19,67s $( \pm 2,6)$ na condição CCSV.

A ANOVA three-way calculada para a duração do deslocamento total (2 condições de tarefa $\mathrm{x} 2$ condições de visão $\mathrm{x} 6$ tentativas) para medidas repetidas em todos os fatores mostrou efeito significativo para a condição de tarefa $\left(\mathrm{F}_{1,12}=\right.$ 23,$\left.33 ; p<0,001 ; \eta^{2} 0,66\right)$, visão $\left(\mathrm{F}_{1,12}=100,92 ; p \leq 0,001 ; \eta^{2}\right.$ $0,89)$ e tentativas $\left(F_{5,60}=8,74 ; p \leq 0,001 ; \eta^{2} 0,42\right)$. A interação significativa entre as condições de tarefa e visão $(p \leq 0,001$; $\left.\eta^{2} 0,76\right)$ indica que os valores médios da duração do deslocamento total não alteraram entre SCCV e CCCV (Figura 1 , lado esquerdo). Interações significativas entre visão e tentativa ( $\left.p=\leq 0,001 ; \eta^{2} 0,39\right)$ indica uma maior duração nas tentativas iniciais em comparação com as finais. A interação tripla entre condição tarefa, visão e tentativa ( $p=0,055 ; \eta^{2}$ $0,16)$ evidencia, através do teste de Bonferroni que as tentativas finais são mais discrepantes (tornam-se mais rápidas) nas condições sem o cão e sem visão ( 1 x 4, 1 x 5, 1 x 6; 2 x 6; 5 x 6) e com o cão e sem visão ( 1 x 5, 1 x 6, 2 x5, 2 x 6, 3 x 5, 3 x 6, 4 × 6) $(p=0,05)$ (Ver Figura 1, lado direito). 

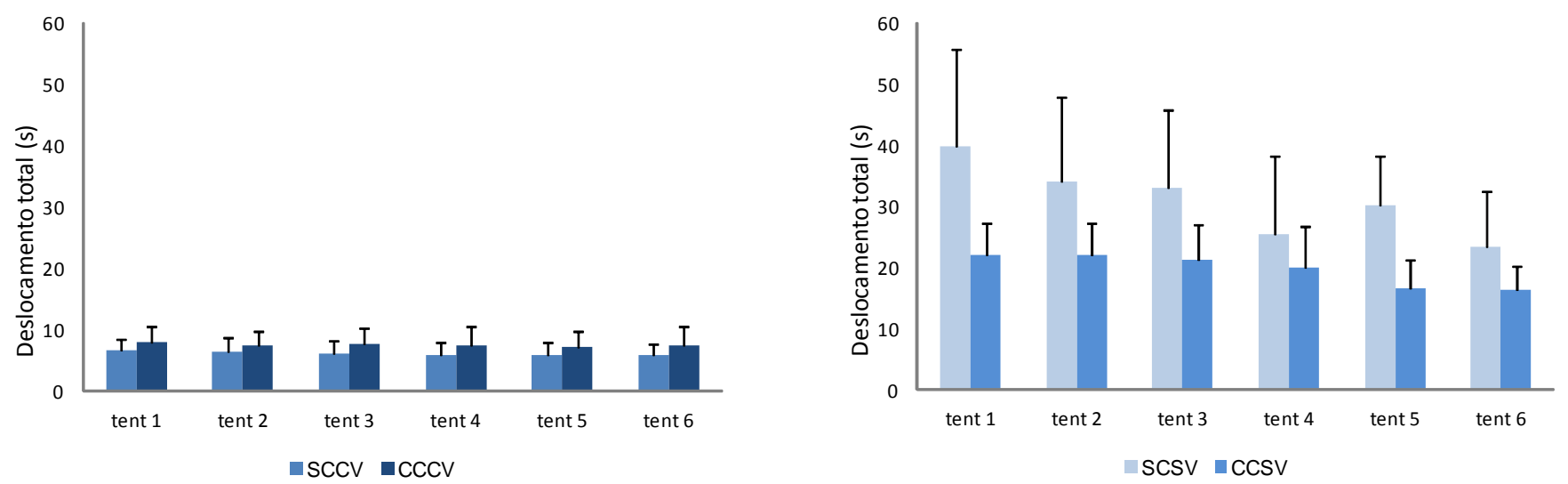

Figura 1. Média e desvio-padrão da duração do deslocamento total nas condições experimentais com e sem o cão, mas com uso da visão (lado esquerdo) e com e sem o cão, mas sem o uso da visão (lado direito).
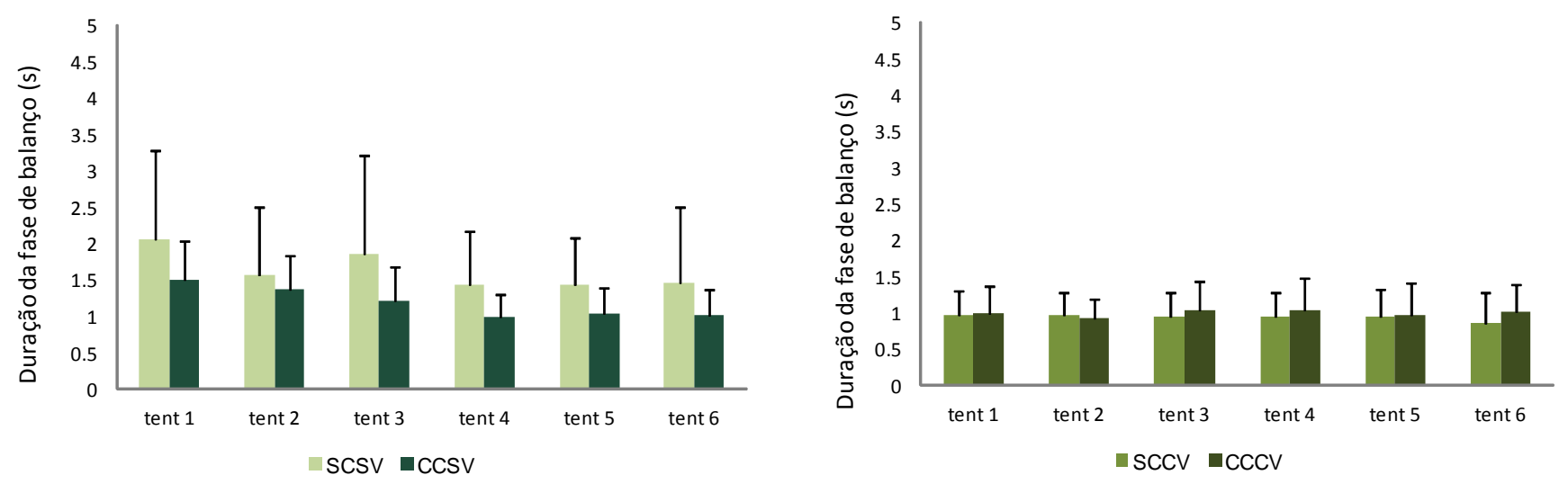

Figura 2. Média e desvio-padrão da duração da fase de balanço nas condições experimentais com e sem o cão, mas com uso da visão (lado direito) e com e sem o cão, mas sem o uso da visão (lado esquerdo).

\section{Duração da fase de balanço}

Os valores da média e do desvio-padrão da fase de balanço dos participantes nas condições experimentais foram: 0,94 s $( \pm 0,04)$ na condição SCCV; 0,99 s $( \pm 0,04)$ na condição CCCV; 1,63 s $( \pm 0,26)$ na condição SCSV e 1,18 s $( \pm 0,2)$ na condição CCSV.

A ANOVA three-way calculada para a duração da fase de balanço (2 condições de tarefa x 2 condições de visão x 6 tentativas) para medidas repetidas em todos os fatores mostrou efeito significativo para a condição tarefa $\left(\mathrm{F}_{1,12}=6,85 ; p\right.$ $\left.=0,022 ; \eta^{2} 0,36\right)$, visão $\left(F_{1,12}=44,18 ; p \leq 0,001 ; \eta^{2} 0,79\right)$. A interação significativa entre as condições de tarefa e visão $\left(p=0,003 ; \eta^{2} 0,54\right)$ aponta para a diferença entre as médias da duração da fase de balanço da condição com cão, com (Figura 2, lado esquerdo) e sem visão (Figura 2, lado direito).

\section{Duração da fase de duplo suporte}

Os valores da média e do desvio-padrão da fase de du- plo suporte dos participantes nas condições experimentais do presente estudo foram: $0,5 \mathrm{~s}( \pm 0,03)$ na condição SCCV; $0,63 s( \pm 0,08)$ na condição CCCV; 2,71 s $( \pm 0,42)$ na condição SCSV e $1,76 s( \pm 0,28)$ na condição CCSV.

A ANOVA three-way calculada para a duração da fase de duplo suporte (2 condições de visão x 2 condições de tarefa x 6 tentativas) para medidas repetidas em todos os fatores mostrou efeito significativo para a condição tarefa $\left(\mathrm{F}_{1,12}=\right.$ 15,$\left.01 ; p=0,002 ; \eta^{2} 0,57\right)$, visão $\left(F_{1,12}=51,25 ; p \leq 0,001 ; \eta^{2}\right.$ $0,81)$. A interação significativa entre as condições de tarefa e visão $\left(p \leq 0,001 ; \eta^{2} 0,63\right)$ aponta para a diferença entre as médias da duração da fase de balanço da condição com cão, com (Figura 3, lado esquerdo) e sem visão (Figura 3, lado direito). Ainda, uma interação significativa observada entre visão e tentativa ( $\left.p=0,006 ; \eta^{2} 0,23\right)$ aponta para redução na duração das passadas, na condição sem visão, para as tentativas finais em comparação com as iniciais. O teste de Bonferroni indicou diferença significativa entre as condições sem cão, sem visão $(1 \times 6)$ e entre as condições com cão, sem visão $(1$ x $2,1 \times 3$, 1 x4 e 1 x 6) $(p=0,05)$. 

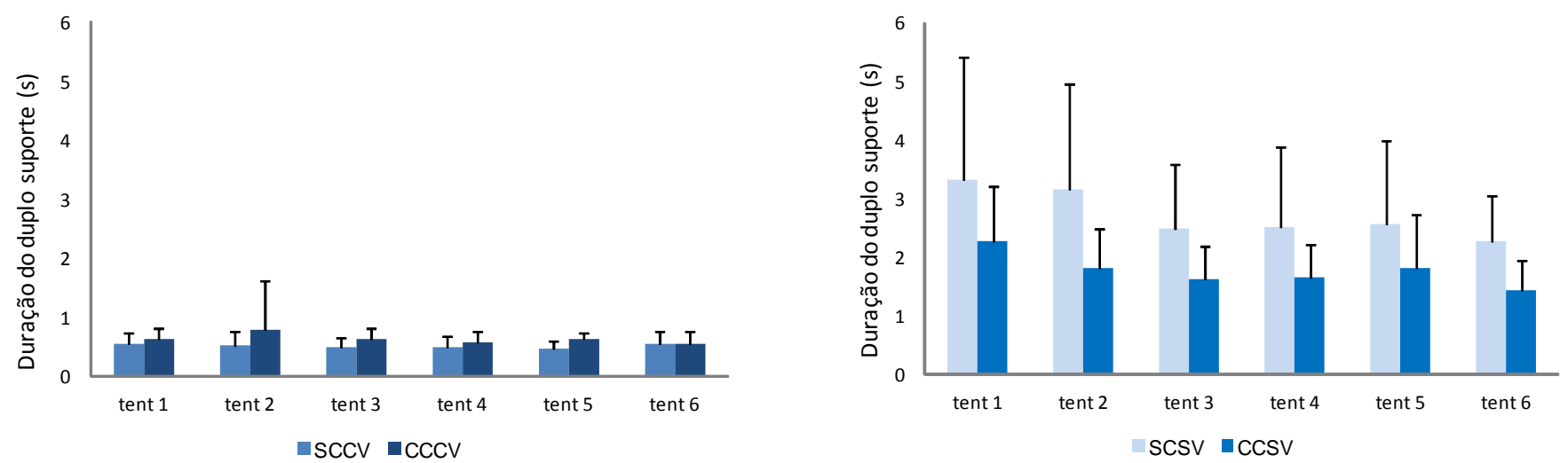

Figura 3. Média e desvio-padrão da duração da fase de duplo suporte nas condições experimentais com e sem o cão, mas com uso da visão (lado esquerdo) e com e sem o cão, mas sem o uso da visão (lado direito).
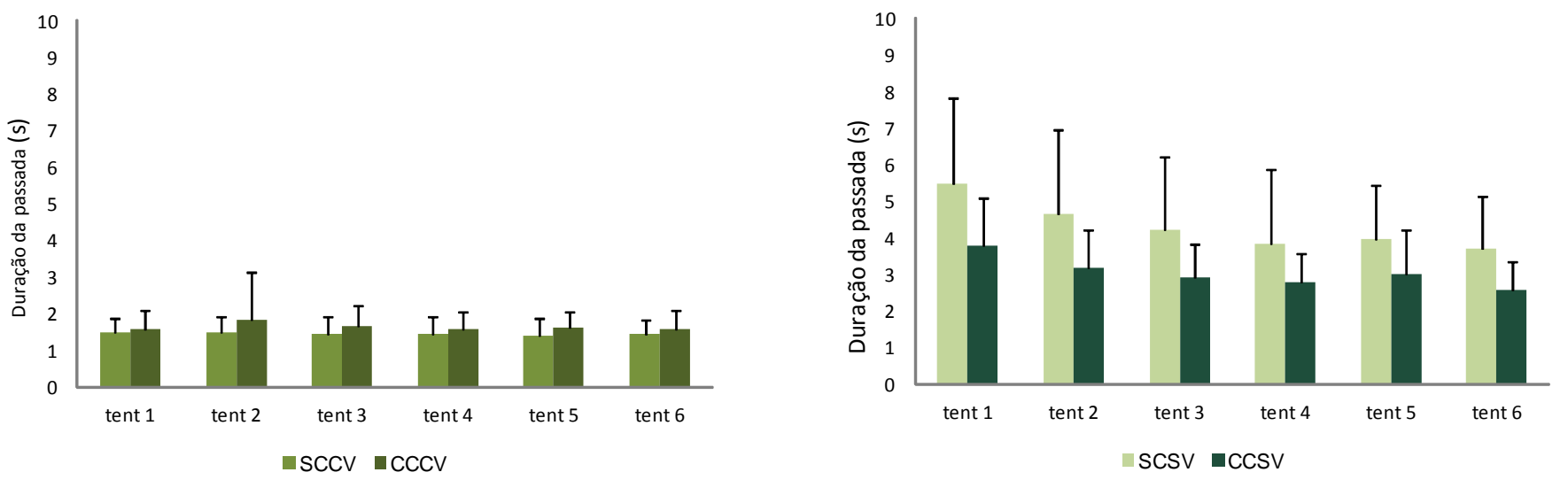

Figura 4. Média e desvio-padrão da duração das passadas nas condições experimentais com e sem o cão, mas com uso da visão (lado esquerdo) e com e sem o cão, mas sem o uso da visão (lado direito).

\section{Duração das passadas}

Os valores da média e do desvio-padrão da duração das passadas dos participantes nas condições experimentais do estudo foram: 1,43 segundos (s) $( \pm 0,02)$ na condição SCCV; $1,63 s( \pm 0,1)$ na condição CCCV; 4,3s $( \pm 0,68)$ na condição SCSV e 3,02s $( \pm 0,42)$ na condição CCSV. A ANOVA threeway calculada para a duração das passadas (2 condições de tarefa x 2 condições de visão x 6 tentativas) para medidas repetidas em todos os fatores mostrou efeito significativo para a tarefa $\left(\mathrm{F}_{1,12}=16,85 ; p \leq 0,001 ; \eta^{2} 0,58\right)$, visão $\left(\mathrm{F}_{1,12}=\right.$ 62,$\left.23 ; p \leq 0,001 ; \eta^{2} 0,88\right)$ e tentativas $\left(\mathrm{F}_{5,60}=4,02 ; p=0,03 ; \eta^{2}\right.$ $0,25)$. A interação significativa entre as condições de tarefa e visão $\left(p \leq 0,001 ; \eta^{2} 0,70\right)$ indica que a similaridade na duração da passada na condição CCCV e SCCV e a interação entre visão e tentativa $\left(p=0,02 ; \eta^{2} 0,27\right)$ aponta para redução na duração das passadas, na condição sem visão, para as tentativas finais em comparação com as iniciais (Figura 4, lado direito). O teste de Bonferroni demonstrou diferença significativa entre as condições sem cão, sem visão (1 x 5 e 1 x6) e entre as condições com cão, sem visão (1 contra todas as tentativas; 2 × 6 e 3 × 6) $(p \leq 0,001)$ (ver Figura 4).

\section{Comprimento das passadas}

A média e o desvio-padrão do comprimento da passada dos participantes nas condições experimentais do presente estudo foram: 114,48 cm $( \pm 2,48)$ na condição SCCV; 106,18 cm $( \pm 2,02)$ na condição CCCV; 65,68 cm $( \pm 0,42)$ na condição SCSV e $1,76 \mathrm{~cm}( \pm 0,28)$ na condição CCSV. ANOVA threeway calculada para o comprimento das passadas (2 condições de visão x 2 condições de tarefa x 6 tentativas) para medidas repetidas em todos os fatores apontou efeito significativo para a condição visão $\left(\mathrm{F}_{1,12}=264,72 ; p \leq 0,001 ; \eta^{2}\right.$ $0,96)$ e tentativas $\left(F_{5,60}=3,32 ; p=0,010 ; \eta^{2} 0,22\right)$. Uma interação significativa entre as condições de tarefa e visão $\left(p=0,003 ; \eta^{2} 0,53\right)$ indica que o comprimento da passada foi similar nas condições CCCV e SCCV (ver Figura 5, lado esquerdo).

\section{Velocidade das passadas}

Os valores da média e do desvio-padrão da velocidade das passadas dos participantes nas condições experimentais do estudo foram: 85,43 cm/s $( \pm 3,06)$ na condição SCCV; 

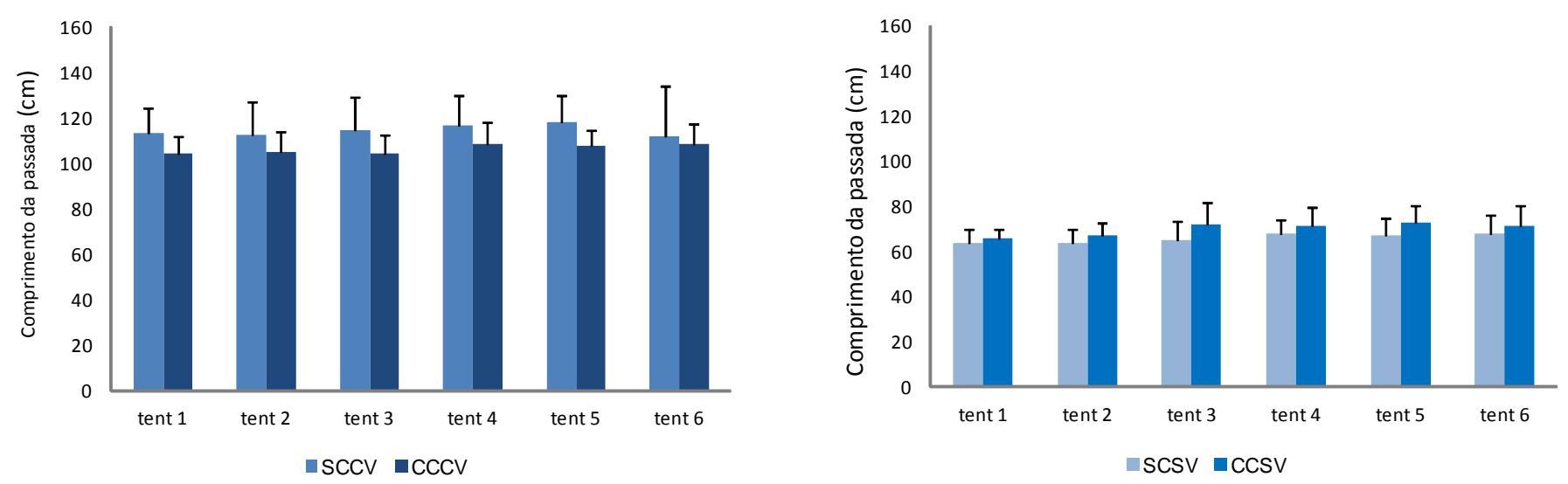

Figura 5. Média e desvio-padrão do comprimento das passadas nas condições experimentais com e sem o cão, mas com uso da visão (lado esquerdo) e com e sem o cão, mas sem o uso da visão (lado direito).
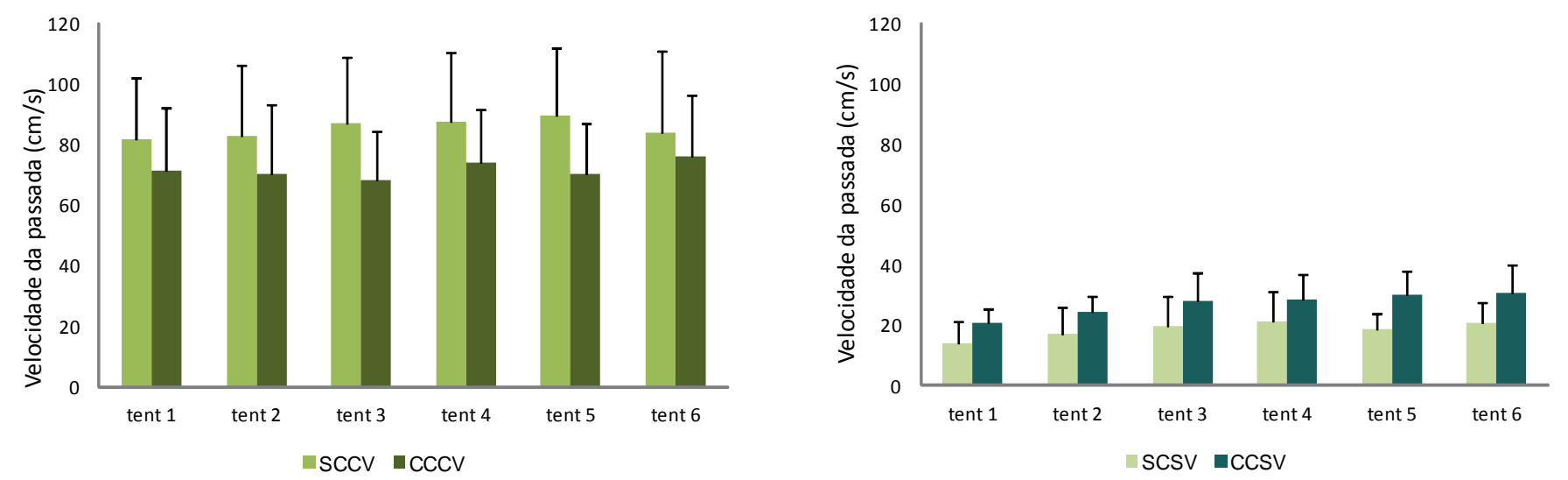

Figura 6. Média e desvio-padrão da velocidade das passadas nas condições experimentais com e sem o cão, mas com uso da visão (lado esquerdo) e com e sem o cão, mas sem o uso da visão (lado direito).

$71,64 \mathrm{~cm} / \mathrm{s}( \pm 2,79)$ na condição CCCV; $18,5 \mathrm{~cm} / \mathrm{s}( \pm 2,7)$ na condição SCSV e 27,09 cm/s $( \pm 3,82)$ na condição CCSV.

ANOVA three-way calculada para a duração da fase de duplo suporte ( 2 condições de visão x 2 condições de tarefa x 6 tentativas) para medidas repetidas em todos os fatores apontou efeito significativo para a condição visão $\left(\mathrm{F}_{1,12}=\right.$ 128,$\left.41 ; p \leq 0,001 ; \eta^{2} 0,91\right)$ e tentativas $\left(\mathrm{F}_{5,60}=4,50 ; p=0,002\right.$; $\left.\eta^{2} 0,27\right)$. Uma interação significativa entre as condições de tarefa e visão $\left(p \leq 0,001 ; \eta^{2} 0,86\right)$ indica que o comprimento da passada foi similar nas condições CCCV e SCCV (ver Figura 6, lado esquerdo).

\section{Fase relativa intermembros}

Os valores da média e do desvio-padrão da fase relativa intermembros nas condições experimentais do estudo foram: $0,51( \pm 0,01)$ na condição SCCV; $0,51( \pm 0,00)$ na condição CCCV (Figura 7, lado esquerdo); $0,51( \pm 0,00)$ na condição SCSV e $0,51( \pm 0,00)$ na condição CCSV (Figura 7, lado direito).

ANOVA three-way calculada para a media da fase relativa de cada condição experimental (2 condições de tarefa x 2 condições de visão x 2 lados) para medidas repetidas em todos os fatores apontou efeito significativo para a condição visão $\left(\mathrm{F}_{1,13}=10,42 ; p=0,007 ; \eta^{2} \quad 0,44\right)$ e para lados $\left(\mathrm{F}_{1,13}\right.$ $=68,87 ; p \leq 0,001 ; \eta^{2} 0,84$ ) (ver Figura 7).

ANOVA three-way calculada para o desvio da fase relativa de cada condição experimental ( 2 condições de tarefa $x$ 2 condições de visão x 2 lados) para medidas repetidas em todos os fatores mostrou efeito significativo para a condição cão $\left(\mathrm{F}_{1,13}=72,76 ; p \leq 0,001 ; \eta^{2} 0,85\right)$ e interação significativa entre lados e cão $\left(p=0,044 ; \eta^{2} 0,23\right)$. A Figura 8 ilustra que na condição sem visão, andar sem o cão causa maior variabilidade de ambas as pernas quando comparada com a condição onde o cão é conduzido (Figura 8, lado direito). Na condição com visão, ambos os lados tendem a mostrar similar variabilidade (Figura 8, lado esquerdo).

\section{Discussão}

O presente estudo investigou a influência da percepção háptica na estabilidade locomotora do ser humano durante a condução de um cão com uma guia. Praticamente todas as variáveis temporais e espaciais do andar do participante 

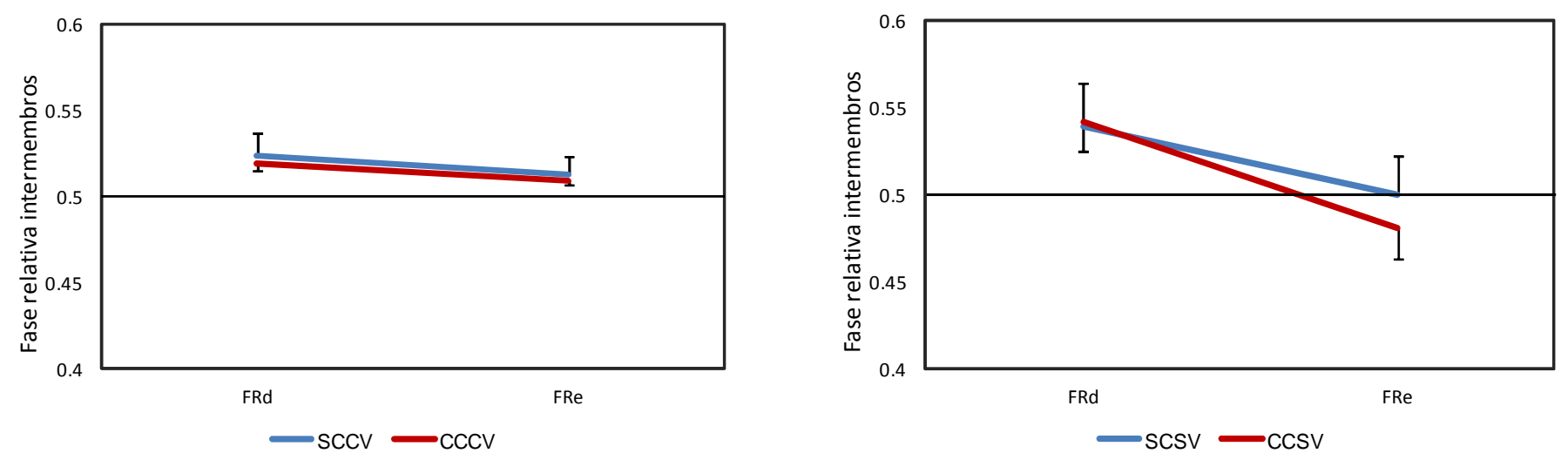

Figura 7. Média e desvio-padrão da fase relativa intermembros nas condições experimentais com e sem o cão, mas com uso da visão (lado esquerdo) e com e sem o cão, mas sem o uso da visão (lado direito).
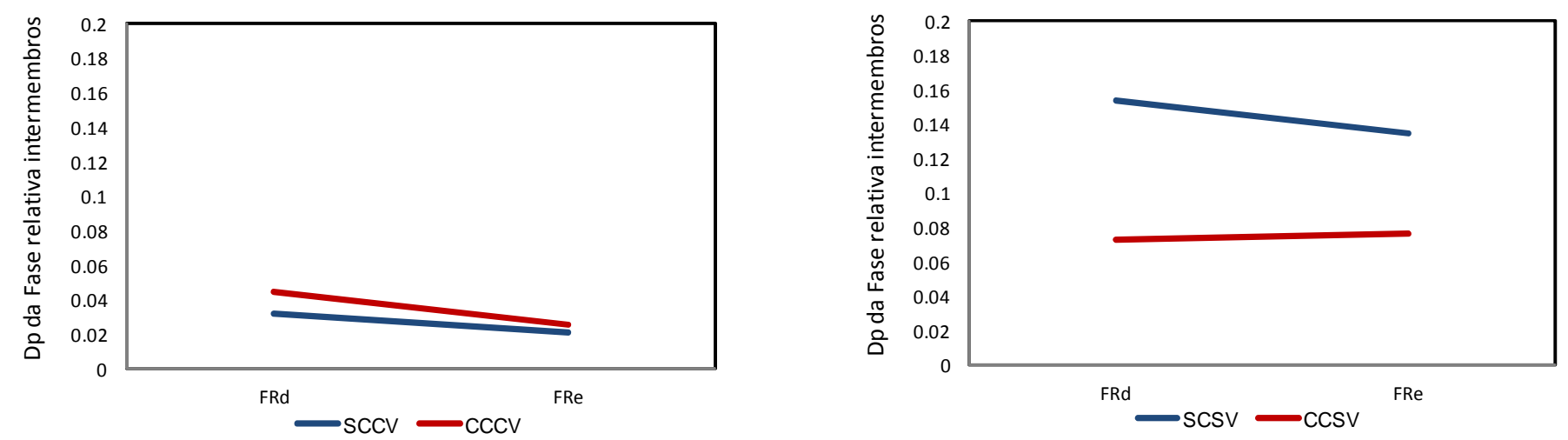

Figura 8. Desvio-padrão (Dp) da fase relativa intermembros nas condições experimentais com e sem o cão, mas com uso da visão (lado esquerdo) e com e sem o cão, mas sem o uso da visão (lado direito).

humano indicaram que, ao conduzir um cão em uma tarefa desafiadora (andar com privação da visão), seus respectivos valores tendem a se aproximar mais daqueles encontrados em condições de andar com a presença da visão, do que quando o andar foi executado de forma independente sem a visão. A partir dos resultados deste estudo pudemos apresentar evidências empíricas de que o uso da guia (ferramenta não rígida), durante uma tarefa com privação visual, auxilia na estabilização locomotora.

Os consistentes resultados das variáveis selecionadas neste estudo confirmam que, ao andar vendado conduzindo o cão, os participantes realizavam a tarefa de forma mais rápida do que quando deslocando sozinhos. Similares resultados também foram observados na duração e amplitude dos ciclos das passadas, confirmando que o movimento locomotor ao longo do trajeto foi feito de forma mais estável e orientada do que quando na situação sem a condução do cão. De fato, a combinação desses elementos resultou num aumento da velocidade de execução dessas passadas. Quanto à variável fase relativa, os valores do desvio-padrão mostraram que a coordenação temporal entre pé direito e esquerdo ao longo das passadas não mudou durante a condução do cão, exceto quando a visão foi obstruída. Neste caso, a variabilidade da fase relativa caiu pela metade e aproximou-se dos valores encontrados na condição com visão. Além da fase relativa confirmar a estabilidade locomotora, permitiu inferir sobre o nível de simetria entre os lados corporais. Um valor igual a 0,5 indica perfeita simetria entre os lados corporais. Neste caso, os valores médios da fase relativa indicaram um aumento de assimetria entre as passadas durante a condução do cão. É possível que a passada de um dos lados corporais tenha sofrido mais impacto do que outro pela tentativa de controlar o animal durante sua condução (feita com a mão direita). Dentre os 14 participantes, apenas um era canhoto.

No modelo experimental deste estudo, observamos que todas as variáveis foram úteis para testar nossa hipótese sobre a possibilidade de efeitos estabilizadores no comportamento locomotor devidos à condução de um cão, particularmente pelos altos valores do parâmetro $\eta^{2}$. De fato, o tamanho do efeito foi alto (acima de 0,50 ) tanto para a condição visão quanto para as interações entre a tarefa com cão e a condição da visão.

Esses resultados dão suporte aos pressupostos do sistema âncora que, em diversos contextos experimentais, confirmou efeitos estabilizadores na postura dos participantes 
(Calve \& Mauerberg-deCastro, 2005; Dascal et al., 2012; Mauerberg-deCastro, 2004; Mauerberg-deCastro et. al., 2012; Melo, 2011; Moraes \& Mauerberg-deCastro, 2009; Polanczyk \& Mauerberg-deCastro, 2005). Assim como naqueles estudos, no presente estudo a informação háptica captada do ambiente foi obtida por meio de uma ferramenta não rígida (i.e., guia do cão) e permitiu melhor estabilidade locomotora durante tarefas com restrições ao equilíbrio. Os resultados do presente estudo também estão de acordo com aqueles obtidos por Melo (2011) na variável duração das passadas e desvio-padrão da fase relativa nas condições de privação visão quando conduzindo um cão numa tarefa locomotora semelhante à do presente estudo.

A não diferença estatística aqui encontrada entre as condições com o uso da visão, em ambas as tarefas com e sem a condução do cão permite inferir que o sistema visual se "encarrega" de propiciar a estabilidade locomotora em demanda. Caminhar junto com o cão e, neste caso, inserir estratégias hápticas para controlar a guia é irrelevante ao sistema postural e à estabilidade locomotora. As condições experimentais sem visão apresentaram exacerbada perturbação postural do ser humano e, por isso, a informação advinda do sistema háptico foi determinante no controle do animal pela guia. De acordo com Bonfim et al. (2006), o uso do sistema háptico é mais requisitado nas condições em que a visão é manipulada de forma a causar perturbação.

Nossos resultados confirmam a predição de que o movimento corporal do cão orientou (através da guia) o sistema postural do condutor durante a tarefa locomotora sem visão. A tensão impressa pelo condutor na guia do cão serviu tanto para controlar o animal, como para fornecer um sistema de referência espacial integrando o cão (e suas reações), a ferramenta em si (a guia) e o meio (superfície e trajeto). Nossa interpretação é de que os dados resultantes desse sistema de referência eram atualizados sempre que uma necessidade postural mais desafiadora se apresentava. A dupla tarefa háptica (i.e., através da guia, controlar o cão e extrair dados sobre a posição do próprio corpo a partir das reações posturais do cão) integrou os dados da tensão na guia sem sacrificar uma ou outra função. Diferentes de outros tipos de tarefas duplas que podem envolver uma tarefa motora (deslocar-se entre obstáculos) simultânea a uma tarefa cognitiva (contar de trás para frente), o sistema âncora parece integrar requerimentos de tarefas posturais de forma cooperativa. O controle do animal com a guia poderia ser abandonado se a guia fosse usada para auxiliar o equilíbrio causando deslocamento do cão. Nossos participantes, baseado em inspeção visual, embora tenham variado a puxada, nunca o fizeram a ponto de deslocar o cão fora da sua superfície de apoio. Neste estudo, a puxada na guia, embora não avaliada em sua magnitude de força tensional, pode ter correspondência com a força tensional aplicada em tarefas de toque leve (Jeka \& Lackner, 1994; 1995).

Os parâmetros temporais representativos do andar (i.e., duração das passadas, das fases de duplo suporte, balanço) foram todos consistentes em mudança conforme a demanda das tarefas. Já a fase relativa intermembros parece mais re- sistente às alterações promovidas pelo contexto da tarefa e, portanto, a variabilidade desta medida tem sido útil em capturar os efeitos de manipulações experimentais (Paula, Mauerberg-deCastro, \& Cozzani, 2006).

O comprimento das passadas nas condições com o uso da visão se assemelhou aos valores médios encontrados na literatura (Newmann, 2011), relação não presente em condições sem o uso da visão. Estes resultados também sugerem que mesmo com a restrição da base suporte, o uso da visão proporcionou informação suficiente aos participantes para atingir estabilidade e orientação postural durante a realização das condições de andar com e sem contato com o cão.

As mudanças no desempenho ao longo das tentativas, especialmente entre a inicial e final apontam para um efeito adaptativo e de aprendizagem ao longo das repetições. Este resultado indica que a repetição pode implicar em benefício do uso do contato mediado com o cão. No estudo de Mauerberg-deCastro et al. (2012), a prática com o sistema âncora por adultos com e sem deficiência intelectual resultou em efeito de curto prazo no controle postural após a remoção do sistema âncora. Entretanto, a prática (i.e., repetições seguidas na mesma taxa dos grupos experimentais) da tarefa postural sem o sistema âncora não resultou nos mesmos benefícios para os grupos controles com e sem deficiência.

O aspecto da repetição também foi investigado em estudo de Freitas et al. (2013). Nele, o uso prolongado do sistema âncora no controle postural de indivíduos idosos foi investigado em diferentes frequências de uso (0, 50, 100\%) durante uma tarefa de controle postural. Apenas o grupo de $50 \%$ mostrou um efeito de retenção na melhora do controle postural após a remoção da ferramenta. O contraste entre demandas ao sistema háptico pela utilização do sistema âncora intervalado com condições sem o mesmo permitiram adaptações imediatas úteis ao sistema postural. Como nossas tentativas foram randomizadas completamente, é possível que o efeito contraste tenha sido propiciado e, dessa forma, a melhora ao longo das repetições tenha decorrido tal como no estudo de Freitas et al. (2013).

A movimentação do cão e a ancoragem através da guia permitiram ao indivíduo captar, reunir e transformar a informação necessária para manter a postura enquanto caminhava. A característica de dupla tarefa foi imposta pela restrição visual permitindo aos participantes manterem a condução do cão ao mesmo tempo em que utilizavam as propriedades hápticas da guia para orientação postural. A eficiência desse uso variou em função da prática apenas quando a necessidade postural foi significativa (i.e., ausência da visão). Por exemplo, o deslocamento total foi mais rápido, assim como a duração das passadas reduziu entre as tentativas iniciais e finais quando a visão não estava disponível. O comprimento e velocidades da passada maiores foram observados nas tentativas finais em todas as condições de tarefas, porém sem significância detectada nas comparações aos pares.

Os resultados do estudo sugerem que os indivíduos são capazes de detectar propriedades hápticas do movimento de um cão por meio da sua guia de modo a atenuar efeitos 
desestabilizadores na locomoção durante contextos de restrição ao equilíbrio e à visão.

As implicações dos efeitos da informação (percepção) háptica no comportamento de indivíduos que interagem, utilizando de mediação por ferramentas tanto com o meio estável (e.g., uma superfície) como com contato com outros organismos, sugere aplicações práticas importantes. Por exemplo, o uso do cão guia como estratégia de navegação e mobilidade do cego amplia o significado de adaptação. Ou seja, é provável que, se a estabilidade postural do cego não for preservada, ou mesmo melhorada, pela utilização do cão guia, a função de navegação poderia ficar prejudicada.

\section{Referências}

Banks, M. R. \& Banks, W. A. (2002). Effects of Animal-Assisted Therapy on Loneliness in an Elderly Population in Long-Term Care Facilities. The Journal of Gerontology, 57(7), 428-432.

Bonfim, T. R., Polastri, P. F. \& Barela, J.A. (2006). Efeito do toque suave e da informação visual no controle da posição em pé de adultos. Revista Brasileira de Educação e Esporte, 20, 15-25.

Burton, G. (1993). Non-neural extensions of haptic sensitivity. Ecological Psychology, 5(2), 105-124.

Calve, T. \& Mauerberg-deCastro, E. (2005). Contribuição da percepção háptica no controle postural de crianças. Motriz, 11(3), 199-204.

Capote, P. S. O. (2009). Terapia assistida por animais (TAA) $e$ deficiência mental: análise do desenvolvimento psicomotor. Dissertação de Mestrado, Programa de Pós-graduação em Educação Especial. Universidade Federal de São Carlos, São Carlos, Brasil.

Carlisle, GK. (2012). Pet dog ownership in families of children with autism: Children's social skills and attachment to their dogs. Doctoral dissertation, graduate program in nursing, University of Missouri. Columbia, United States.

Cohen, S. (2004). Social relationships and health. American Psychologist, 59, 676-684.

Cutt, H., Giles-Corti, B., Knuiman, M., \& Pikora, T. (2008). Physical activity behavior of dog owners: development and reliability of the Dogs And Physical Activity (DAPA) tool. Journal of Physical Activity \& Health, 5, 73-89.

Dascal, J. B.; Okazaki, V.H.A., \& Mauerberg-deCastro, E. (2012). Efeitos do sistema âncora sobre o controle postural de idosos. Revista Brasileira de Cineantropometria e Desempenho Humano, 14(2):144-153. DOI: http://dx.doi.org/10.5007/19800037.2012v14n2p144

Figueroa, P. J., Leite, N. J., \& Barros, R. M. (2003). A flexible software for tracking of markers used in human motion analysis. Computer methods and programs in biomedicine, 72(2), 155165.

Freitas, M. B. Z., Mauerberg-deCastro, E., \& Moraes, R. (2013). Intermittent use of an "anchor system" improves postural control in healthy older adults. Gait Posture. doi: 10.1016/ j.gaitpost.2013.01.004

Friedmann, E., Thomas S. A., Stein, P.K., \& Kleiger, R.E. (2003). Relationship between pet ownership and heart rate variability in patients with healed myocardial infarcts. The American Journal of Cardiology, 91(6), 718-721.

Gee, N. R., Harris, S. L., \& Johnson, K. L. (2007). "The role of therapy dogs in speed and accuracy to complete motor skills tasks for preschool children. Anthrozoos, 20 (4), 375-386.

Gentaz, E.; Baud-Bovy, G., \& Luyat, M. (2008). The haptic perception of spatial orientations. Experimental Brain Research, 187(3): 331-348. doi: 10.1007/s00221-008-1382-0

Grandgeorge, M., Tordjman, S., Lazartigues, A., Lemonnier, E., Deleau, M., \& Hausberger, M. (2012). Does pet arrival trigger prosocial behaviors in individuals with autism? Plus one. doi:10.1371/journal.pone.0041739.

Gibson, J.J. (1962). Observation on active touch. Psychology Review, 69, 477-491.

Jalongo, M. R., Astorino, T., \& Bomboy, N. (2004). Canine Visitors: The Influence of Therapy Dogs on Young Children's Learning and Well-Being in Classrooms and Hospitals. Early Childhood Educational Journal, 32, 9-16.

Jeka, J.J., \& Lackner, J.R. (1994). Fingertip contact influences human postural control. Experimental Brain Research, 100(3), 495-502.

Jeka, J.J., \& Lackner, J.R. (1995) .The role of haptic cues from rough and slippery surfaces on human postural control. Experimental Brain Research, 103(2): 267-276.

Johnson, R.A., \& Meadows, R.L. (2010). Dog-Walking: Motivation for adherence to a walking program. Clinical Nursing Research, 19(4), 387-402.

Katcher, A.H., \& Teumer, S.P. (2006). A 4-year trial of animalassisted therapy with public school special education students. In A.H. Fine (Ed.), Handbook on Animal-Assisted Therapy: Theoretical Foundations and Guidelines for Practice $\left(2{ }^{\text {nd }} E d\right.$., pp. 227-242). Burlington: Elsevier.

Kushner, R. F., Blatner, D. J., Jewell, D. E., \& Rudloff, K. (2006). The PPET study: People and pets exercising together. Obesity, 14(10), 1762-1770.

Martin, F., \& Farnum, J. (2002). Animal-assisted therapy for children with pervasive developmental disorders. Western Journal of Nursing Research, 24(6), 657-670.

Mauerberg-deCastro, E., \& Angulo-Kinzler, R. (2001). Vantagens e limitações das ferramentas usadas para investigar padrões de comportamento motor segundo a abordagem dos sistemas dinâmicos. In: L.A. Teixeira. Avanços em comportamento motor (Ed) (pp 35-57). São Paulo: Movimento.

Mauerberg-deCastro, E.; Lucena, C.S.; Cuba, B.W.; Boni, R.C.; Campbell, D.F., \& Moraes, R. (2010). Haptic Stabilization of Posture in Adults With Intellectual Disabilities Using a Nonrigid Tool. Adapted Physical Activity Quarterly, 27, 208-225.

Mauerberg-deCastro, E. (2004). Developing an "anchor" system to enhance postural control. Motor control, 8(3), 339-358.

Mauerberg-deCastro, E., Moraes, R., \& Campbell, D. F. (2012) Short-term effects of the use of non-rigid tools for postural control by adults with intellectual disabilities. Motor control, 16(2), 131-143.

Mauerberg-deCastro, E.; Moraes, R.; Tavares, C. P.; Pestana, M.B.; Porto, L.A., \& Dias, M.S. (2013). Utilidade da informação háptica para indivíduos em uma posição de equilíbrio estática conectados a um cão andando em uma esteira rolante. Relatório de pesquisa. Universidade Estadual Paulista.

Melo, J. C. (2011). A ancoragem funcional através do manejo de cão em tarefas de equilíbrio. Dissertação de Mestrado, Programa de Pós-graduação em Ciências da Motricidade. Universidade Estadual Paulista, Rio Claro, Brasil.

Moraes, R., \& Mauerberg-deCastro, E. (2009). O uso de ferramenta não-rígida reduz a oscilação corporal em indivíduos idosos. Motriz, 15(2), 263-272.

Newmann, D. A. (2011). Cinesiologia do aparelho musculoesquelético: fundamentos para a reabilitação física ( $2^{\mathrm{a}}$ ed.). Rio de Janeiro: Guanabara Koogan. 
Paula, A.I., Mauerberg-deCastro, E., \& Cozzani, M. V. (2006). Desenvolvimento longitudinal do andar para frente e para trás: Impacto da restrição ambiental. Revista Brasileira de Cineantropometria \& Desempenho Humano, 8(4), 73-81.

Pestana, M. B., Mauerberg-deCastro, E., Périco, B. C., \& Porto, L. A. (2012). Percepção háptica durante a condução de um cão. In: Trabalhos do XXIV Congresso de Iniciação Científica da UNESP. XXIV Congresso de Iniciação Científica da UNESP São Pedro 2012 São Paulo: PROPE. http://prope.unesp.br/ xxiii_cic/fase1.php

Polanczyk, S. D., \& Mauerberg-deCastro, E. (2005). Contribuição do paradigma âncora na melhora do controle postural em adultos com deficiência mental após um treinamento. Motriz, 117(Supl.1), S141-S142.

Serpell, J.A. (2004). Factors influencing human attitudes to animals and their welfare. Animal Welfare, 13, 145-151.

Tardona, D. R. (2012). Promoting Companion Animal Leash Compliance on an Urban Park Trail System. Natural Areas Journal, 32(2), 215-217.

Thalheimer, W., \& Cook, S. (2002). How to calculate effect sizes from published research articles: A simplified methodology. http://work-learning.com/effect_sizes.htm.

Toohey, A.M., \& Rock, M.J. (2011). Unleashing their potential: a critical realist scoping review of the influence of dogs on physical activity for dog-owners and non-owners. International Journal of Behavioral Nutrition and Physical Activity. doi:10.1186/1479-5868-8-46

Zabihaylo, C., Couturier, J.A., Termoz, N., \& Prince, F. (2005). Analysing the postural and gait behaviour of a person with retinitis pigmentosa traveling with a guide dog. International Congress Series, 1282, 767-771.

\section{Nota dos autores}

Eliane Mauerberg-deCastro é professora adjunta na Universidade Estadual Paulista, campus de Rio Claro. Coordena o Laboratório de Ação e Percepção na unidade.

Bruna Carla Périco e Stephanie C. Miranda Pacheco são mestrandas do programa de pós-graduação de Ciências da Motricidade da Universidade Estadual Paulista, campus de Rio Claro. Parte deste trabalho refere-se a dissertação de mestrado de Bruna Carla Périco.

Mayara Borkowske Pestana, Larissa A. Porto e Fernanda L. Magre são alunas de graduação em educacação física, as duas primeiras são bolsistas CNPq-PIBIC e a última bolsista PROEX-UNESP.

\section{Agradecimentos}

CNPq-PIBIC, CAPES e FAPESP.

Agradecemos os comentários e correções de revisores anônimos.

\section{Correspondência}

Eliane Mauerberg-deCastro

Universidade Estadual Paulista

Instituto de Biociências

Av. 24-A, 1515, Bela Vista

Rio Claro SP 13506-900

Email: mauerber@rc.unesp.br

Artigo completo de trabalho apresentado no VIII Congresso Internacional de Educação Física e Motricidade Humana e XIV Simpósio Paulista de Educação Física.

Declaração de Conflito de Interesses: Os autores declararam ausência de conflitos de interesse no que diz respeito à pesquisa, autoria e/ou publicação deste artigo. Para evitar conflito de interesses, a revisão deste manuscrito foi coordenada pela editora associada da Revista Motriz, Profa. Dra. Cynthia Y. Hiraga.

Manuscrito recebido em 18 de março de 2013

Manuscrito aceito em 13 de maio de 2013 